\title{
Bridging the Gap - an Update of Translational Research in Breast Cancer
}

\author{
Cornelia Liedtke ${ }^{a}$ Ludwig Kiesel $^{b}$ \\ aKlinik für Frauenheilkunde und Geburtshilfe, Universitätsklinikum Schleswig-Holstein / Campus Lübeck, \\ ${ }^{b}$ Klinik für Frauenheilkunde und Geburtshilfe, Universitätsklinikum Münster, Germany
}

High-throughput gene expression and mutation analysis have provided increasing insight into the complex molecular biology of breast cancer. For many decades, detailed knowledge of the basic molecular biology of breast cancer did not have significant impact on routine clinical management of the disease. However, with increasing insights into both breast cancer molecular heterogeneity and targetable signal transduction pathways, scientists as well as clinicians have to understand the increasing complexity of the disease to be able to offer an individualized treatment strategy to patients with breast cancer. Molecular biology becomes relevant for treatment selection, understanding efficacy, side effects, and resistance as well as for generating new hypotheses for combinations of therapeutic agents.

While 10 years ago, Perou et al. [1] have suggested breast cancer to be divided into 5 distinct molecular subtypes, it has recently been suggested that each breast cancer subtype is far from being homogeneous. For instance, triple negative breast cancer (TNBC) is understood to consist of several subtypes, presumably each with different prognostic and predictive implications. In order to understand recent developments in breast cancer molecular biology, it is of importance to have basic knowledge of the underlying technologies. In this special issue of Breast Care, Thomas Karn gives insights into the recent development of high-throughput gene expression and mutation profiling [2]. He provides an overview of the technologies used and their applicability in basic, translational, and clinical breast cancer research.

Using these modern technologies, breast cancer has been shown to be comprised of several subtypes. Using complex gene expression profiling methods, basal-like, HER2-like, luminal, and presumably normal-like breast cancer subclasses may be assigned. These subgroups show significant overlap with the clinically defined triple-negative, HER2-positive and hormone receptor-positive breast cancer subtypes, however, it is important to recognize that there may be significant discordance between both classification systems. Both classifications show significant differences with regard to prognosis, response to (chemo-) therapy and underlying molecular biology. In this special issue of BREAST CARE, 2 breast cancer subtypes will be portrayed in detail. First, Gluz et al. [3], summarize the current data regarding hormone receptor-positive breast cancer. The most important clinical question for patients with hormone receptor-positive breast cancer is to identify those patients that have an excellent prognosis after being treated with endocrine therapy alone (usually referred to as luminal-A disease). In contrast, there is a subgroup of patients with highly proliferative, partially endocrine resistant disease that would additionally need (chemo)therapy. The authors summarize the current profiling technologies including immunohistochemical tools and gene expression profiling methods. The authors underscore, however, that current guidelines in Germany suggest only limited use of these tools in selected patients and, if possible, as part of clinical trials.

TNBC is defined by an absence of hormone receptor expression and lack of HER2 overexpression / amplification. There is significant overlap between TNBC and the basal-like breast cancer subtype which is usually defined through complex gene expression profiling or a panel of (immunohistochemical) surrogate markers. In contrast to luminal breast cancer where major efforts are being made to identify patients that do not need chemotherapy with the goal of reducing chemotherapeutic frequency, patients with TNBC suffer from an unfavorable prognosis which is largely due to a lack of therapeutic agents. Therefore, there is an increasing need to identify promising therapeutic targets for patients with TNBC. Liedtke et al. [4] describe the efforts being made in this context. The authors summarize the current knowledge regarding the molecular biology of TNBC including the association with basal-like breast cancer and hereditary breast cancer with a particular focus regarding mutational and gene expression profiling methods and the association between TNBC and breast cancer stem cells.

\section{Disclosure Statement}

The authors have no conflicts of interest relevant to this manuscript to disclose.

\section{KARGER \\ Fax +497614520714}

Information@Karger.com

www.karger.com (c) 2013 S. Karger GmbH, Freiburg

$1661-3791 / 13 / 0086-0397 \$ 38.00 / 0$

Accessible online at:

www.karger.com/brc 


\section{References}

1 Perou CM; Sørlie T; Eisen MB, et al.: Molecular portraits of human breast tumours. Nature 2000;406:747-752.

2 Karn T: High-throughput gene expression and mutation profiling: current methods and future perspectives. Breast Care 2013;8: DOI: 10.1159/000357461.
3 Gluz O, Hofmann D, Würstlein R, Liedtke C, Nitz U, Harbeck N: Genomic profiling in luminal breast cancer. Breast Care 2013;8: DOI: 10.1159/000357535.

4 Liedtke C, Bernemann C, Kiesel L, Rody A: Genomic profiling in triple-negative breast cancer. Breast Care 2013;8: DOI: 10.1159/000357534. 